\section{Krebsbekämpfung: Fortschritte überwiegen}

\author{
Der Kampf gegen den Krebs - bei welchen Entitäten wurden Fortschritte \\ erzielt, wo gab es Verschlechterungen? Eine epidemiologische Untersuchung \\ aus den Niederlanden zeigt die wichtigsten Trends.
}

\begin{abstract}
nformationen über Fortschritte in der Krebsbekämpfung werden häufig allein aus Überlebensstatistiken gewonnen. In der Untersuchung einer niederländischen Arbeitsgruppe wurden Überlebensdaten zusammen mit Daten zu Inzidenz und Mortalität ausgewertet, um belastbarere Aussagen treffen zu können. Ausgewertet wurden dazu Daten aus verschiedenen niederländischen Registern von 1989 bis 2009. Optimaler Fortschritt in der Bekämpfung der Krebsentität wurde definiert als Absinken der Inzidenz und/oder einer Verbesserung beim Überleben begleitet von einer abnehmenden Mortalität. Ein Fortschritt wurde angenommen bei abnehmender Inzidenz und/oder verbessertem Überleben ohne Abnahme der Mortalität, eine Ver-
\end{abstract}

schlechterung bei steigender Inzidenz und/oder verschlechtertem Überleben begleitet von steigender Mortalität.

Bei Männern ergab sich ein optimaler Fortschritt bei zwölf von 19 Krebstypen: Malignomen von Speiseröhre, Lunge, Magen, Gallenblase, Kolon, Rektum, Blase, Prostata und Schilddrüse sowie Leukämie, Hodgkin- und Non-Hodgkin-Lymphomen. Bei Frauen war ein optimaler Fortschritt bei zwölf von 21 Krebsarten zu verzeichnen: Magen, Gallenblase, Kolon, Rektum, Brust, Zervix, Uterus, Ovar und Schilddrüse sowie ebenfalls Leukämie, Hodgkin- und Non-Hodgkin-Lymphomen. Beim malignen Melanom zeigten sich sowohl bei Männern als bei Frauen Verschlechterungen, bei Männern zusätzlich beim
Ösophagus- und Nierenkrebs, bei den Frauen zusätzlich bei Krebs der Mundhöhle, des Rachens, der Speiseröhre, von Pankreas und der Lunge. Die Verschlechterung bei den letztgenannten Entitäten bei Frauen dürfte vor allem auf den seit Anfang der 70er Jahre gestiegenen Zigaretten- und Alkoholkonsum zurückzuführen sein.

Fazit: In den Niederlanden wurden bei den meisten Krebsentitäten seit Ende der 80er Jahre Fortschritte bei Inzidenz und/ oder Überleben erzielt. Verschlechtert hat sich die Situation beim Melanom, bei Frauen zusätzlich bei Tumoren von Mund, Rachen, Speiseröhre und Lunge. Die Überlebensprognose bei Karzinomen der Lunge sowie von Gallenblase, Pankreas und Magen bleibt weiterhin schlecht.

Barbara Kreutzkamp

Karim-Kos HE et al. Progress against cancer in the Netherlands since the late 1980s: An epidemiological evaluation. Int J Cancer. 2012; 130(12):2981-9.

\title{
Das Überleben von HIV-Patienten mit Krebs
}

Wer sich mit dem Aids-Erreger ansteckt, kann heute dank intensiver antiretroviraler Therapien noch lange leben. Dafür ist Krebs inzwischen die häufigste Todesursahe bei HIV-Patienten. Wie gut sind ihre Überlebenschancen?

eit Einführung der hochaktiven ant$\checkmark$ riretroviralen Therapie (HAART) in den 1990er-Jahren ist die Mortalität von HIV-Infizierten dramatisch zurückgegangen, die krebsbedingte Mortalität aber gestiegen. Heute ist Krebs die häufigste Todesursache bei HIV-Infizierten. Um Daten zu ihren Überlebenschancen zu erfassen, durchforsteten Wissenschaftler aus Mailand ihre Archive.

Die Daten der monozentrischen retrospektiven Beobachtungsstudie wurden zwischen Januar 1991 und April 2010 erhoben. Von 6.495 Personen mit HIV-Infektion entwickelten 615 (9,5\%) später noch eine Krebserkrankung, davon 431 (70\%) Tumoren, die nach den Kriterien der Centers of Disease Control als „Aidsdefining" gelten wie Kaposi-Sarkom, Non-Hodgkin-Lymphom und Zervixkarzinom. Die übrigen 184 (30\%) entwickelten einen Krebs, der üblicherweise nicht direkt mit Aids in Zusammenhang steht wie Hodgkin-Lymphom, Bronchialkarzinom oder Leberkrebs.

Die Überlebenschancen der Patienten mit Aids-definierenden Malignomen waren signifikant günstiger, wenn der Krebs erst nach Beginn der HAART-Ära, also nach 1998, diagnostiziert wurde: Die 10-Jahres-Überlebensrate lag dann bei 43,2\%. Wurde das Malignom vorher entdeckt, betrug sie nur $16,4 \%$. Einen ähnlichen Unterschied gab es auch für die nicht mit Aids in Zusammenhang stehenden Tumoren: 44,7\% nach bzw. $33,3 \%$ vor HAART.

Wenn nach der Art des Krebses unterschieden wurde, ergab sich ein ähnliches Bild: Beim Non-Hodgkin-Lymphom steigerte sich die 10-Jahres-Überlebensrate von $11,4 \%$ in der Prä-HAART-Ära auf $42,1 \%$ in der HAART-Ära, beim Kaposi-Sarkom von $23,5 \%$ auf $44,0 \%$, beim Hodgkin-Lymphom von 40,0\% auf $49,5 \%$.
Die Überlebenschancen der HIV-Patienten mit manchen Krebsarten liegen demnach auch mit HAART noch deutlich unter denen der Allgemeinbevölkerung: Den Eurocare-4-Daten zufolge kann bei einem Hodgkin-Lymphom üblicherweise von einer 10-Jahres-Überlebensrate von $76,7 \%$ ausgegangen werden (statt 49,5\% mit HIV); beim Lungenkrebs von 9,6\% (statt $0 \%$ mit HIV).

Fazit: Auch mit der HAART-Therapie sind die Überlebenschancen von HIVPatienten, die Krebs entwickeln, insgesamt gering. Dies ist zum Teil auf ihre Komorbiditäten (wie Hepatitis) zurückzuführen, zum Teil auch auf ein aggressiveres Verhalten des Krebses in dem geschwächten Körper und zum Teil auf ein weiter fortgeschrittenes Stadium der Tumorerkrankung zum Zeitpunkt der Diagnose. Weitere Forschung, wie dem Krebs in diesem besonderen Kontext zu begegnen ist, sind nötig. Christina Berndt

Spagnuolo V et al. Ten-year survival among HIV1 -infected subjects with AIDS or non-AIDS-defining malignancies. Int J Cancer. 2012;130(12): 2990-6. 